\title{
Desafios na transição dos atendimentos psicoterápicos presenciais para online na pandemia de COVID-19: revisão sistemática
}

\author{
Challenges in the transition from face-to-face to online psychotherapeutic care in \\ the COVID-19 pandemic: systematic review

\section{Desafíos en la transición de la atención psicoterapéutica presencial a la atención online en la pandemia de COVID-19: revisión sistemática}

Tatiele Jacques Bossi $\mathbb{D}^{\mathrm{a}}$, Indianara Sehaparini (iD)

a Universidade Federal do Rio Grande do Sul, Programa de Pós-Graduação em Psicologia - Porto Alegre/RS - Brasil.

Instituição: Universidade Federal do Rio Grande do Sul

DOI 10.5935/2318-0404.20210012

\section{Resumo}

Este estudo teve como objetivo investigar os desafios para as psicoterapias na transição dos atendimentos presenciais para online durante a pandemia de COVID-19, através de uma revisão sistemática da literatura. A busca pelo material consultado se deu em bases de dados científicas por meio da combinação de descritores sobre o tema, cujo levantamento apontou 7 estudos publicados no contexto internacional no ano de 2020. Os resultados mostram que a videoconferência foi a Tecnologia de Informação e Comunicação mais utilizada. A transição dos atendimentos presenciais para online impuseram diversos desafios aos psicoterapeutas e pacientes, uma vez que exigiu de ambas as partes encontrar um ambiente adequado em casa para os atendimentos. Além disso, os desafios nesse processo de transição estiveram relacionados a aspectos da interação terapêutica, como dificuldades em se conectar aos pacientes e em ler suas emoções. A adaptação ao novo ritmo da sessão no contexto online, diferenciado do atendimento presencial, também foi destacado. Evidencia-se a predominância de pesquisas relacionadas à psicoterapia online adulta e discute-se a necessidade de estudos sobre o manejo e a efetividade da psicoterapia online com crianças e adolescentes. Conclui-se que o aprimoramento do psicólogo referente ao trabalho clínico online torna-se uma necessidade emergente.

Palavras-chaves: Psicoterapia; Tecnologia da informação; Coronavírus 


\section{Abstract}

This study aimed to investigate the challenges for psychotherapies in the transition from face-to-face to online care during the COVID-19 pandemic, through a systematic review. The search for the consulted material was made in scientific databases through a combination of descriptors about the topic, whose survey showed 7 studies published in internationally context in 2020. The results show that videoconferencing presented itself as the most used Information and Communication Technology. The transition from in-person to online care posed several challenges for psychotherapists and patients, as it required both parties to find a suitable home environment for care. In addition, challenges in this transition process were related to aspects of therapeutic interaction, such as difficulties in connecting with patients and reading their emotions. Adapting to a new rhythm of the session in online context, differentiated from the face-to-face care, was also highlighted. The predominance of research related to adult online psychotherapy is evidenced, and the need to advance studies on handling and effectiveness of online psychotherapy with children and adolescents is discussed. We conclude that the need for psychologist's improvement regarding online clinical work becomes an emerging need.

Keywords: Psychotherapy; Information Technology; coronavirus

\section{Resumen}

Este estudio tuvo como objetivo investigar los desafíos para las psicoterapias en la transición de la atención presencial a la online durante la pandemia de COVID-19 a través de una revisión sistemática. La búsqueda del material consultado se realizó em bases de datos científicas mediante la combinación de descriptores sobre el tema, cuya encuesta apuntó a 7 estudios publicados em el contexto internacional en el año 2020. Los resultados indican que la videoconferencia se presentó como la Tecnología de la Información y la Comunicación más utilizada. La transición de la atención presencial a la online planteó varios desafíos para psicoterapeutas y pacientes, ya que requería que ambas partes encontraran un entorno doméstico adecuado para la atención. Además, los desafíos en este proceso de transición estaban relacionados con aspectos de la interacción terapéutica, como dificultades para conectarse con los pacientes y leer sus emociones. También se destacó la adaptación al nuevo ritmo de la sesión en el contexto online, diferenciado del servicio presencial. Es evidente el predominio de la investigación relacionada con la psicoterapia online de adultos y se discute la necesidad de avanzar en los estudios sobre el manejo y efectividad de la psicoterapia online con niños y adolescentes. Se concluye que la necesidad de perfeccionamiento del psicólogo respecto al trabajo clínico online se convierte en una necesidad emergente.

Palabras clave: Psicoterapia; Tecnología de la información; Coronavirus

\section{Introdução}

A pandemia de COVID-19 foi declarada pela Organização Mundial da Saúde (OMS) no dia 11 de março de 2020, devido à rápida disseminação mundial do vírus Sars-Cov-2, identificado pela primeira vez em 
dezembro de 2019. Medidas de isolamento social foram tomadas, a fim de amenizar a propagação do vírus e, consequentemente, sua taxa de contaminação e letalidade. Frente a isso, diversas transformações pessoais e laborais precisaram ocorrer, inclusive na vida dos psicólogos e de seus pacientes. A necessidade de adaptação à psicoterapêutica mediada pelas Tecnologias de Informação e Comunicação (TIC) foi uma urgência que trouxe à tona diversos desafios para os profissionais frente a realização dos atendimentos online ${ }^{1}$.

Apesar de ser uma prática que existe desde a década de 1950, os atendimentos psicológicos por meio das TICs só começaram a ser inseridos no Brasil nas duas últimas décadas². O Conselho Federal de Psicologia (CFP) autorizou, no ano de 2005, as psicoterapias online por meios síncronos (videoconferência e telefone) ou assíncronos (mensagens de texto e e-mails) apenas para fins de pesquisa, com o propósito de obter resultados científicos sobre a sua efetividade. Já no ano de 2012, houve a ampliação para a oferta de atendimento online breve $^{2}$. Somente no ano de 2018, a psicoterapia online passou a ser autorizada como prática do psicólogo, tendo como exigências o registro profissional e o "Cadastro e-Psi", que se refere a uma autorização, emitida pelo CFP, para que o profissional possa realizar atendimentos psicológicos diversos mediados pelas TICs ${ }^{1}$.

Debates sobre a necessidade de capacitação para o atendimento psicológico online já ocorriam desde antes da regulamentação da prática. Questões sobre a eficácia do tratamento e a dificuldade na construção do setting terapêutico no ambiente online estavam em voga com o intuito de preparar os profissionais e os pacientes para os atendimentos ${ }^{3}$. Assim, a pandemia de COVID-19 veio trazer enormes desafios, ao se considerar a pouca familiaridade dos profissionais com a atuação psicoterapêutica online. Dessa forma, o objetivo deste estudo é investigar os desafios para as psicoterapias na transição dos atendimentos presenciais para online durante a pandemia de COVID-19.

\section{Método}

Esta é uma comunicação breve, em que se realizou uma revisão sistemática da literatura sobre psicoterapias online no contexto da pandemia de COVID-19. A busca pelo material consultado se deu nas bases de dados Scientific Eletronic Library Online - SciELO, PubMed e PsycINFO, por meio dos descritores "(online psychotherapy OR online psychological interventions) and (COVID-19 OR coronavirus)", e selecionou-se como período de busca o ano de 2020 (seleção do material realizada entre 16 e 24 de fevereiro de 2021).

Ao todo, foram encontrados 380 documentos, distribuídos entre as bases de dados: nenhum estudo na SciELO, 265 na PubMed e 115 na PsycINFO. Os artigos encontrados foram classificados a partir dos critérios de inclusão: a) ser estudo empírico; e, b) ter como foco do estudo o atendimento psicológico online e a pandemia de COVID-19. Foram excluídas aquelas referências repetidas entre as bases de dados ( $n=43)$, revisões de literatura $(n=59)$, outros tipos de documentos como cartas ao editor, editorial, debates, entrevistas, comentários e relatos de experiência ( $n=28$ ). Além disso foi excluído um estudo que não apresentava o texto completo. Por fim, foram excluídos os artigos que não se referiam à temática em estudo $(n=219)$. A partir disso, restaram 30 estudos que abordavam sobre o atendimento psicológico online e a pandemia de COVID-19. Para fins da presente comunicação breve, foram selecionados apenas os estudos sobre psicoterapia online e COVID-19, sendo 
excluídos aqueles sobre outras formas de intervenção (ex: intervenção breve e protocolos de atendimento). Com isso, restaram para a análise sete estudos, que foram lidos na íntegra, e estão apresentados na Tabela 1.

\section{Resultados e discussão}

Nos estudos analisados sobre psicoterapia online, a videoconferência foi a Tecnologia de Informação e Comunicação mais utilizada ${ }^{4,5,6,7,8}$. Pode-se pensar que a preferência por chamadas de vídeos se deve ao fato de que possibilita, de certo modo, a reprodução de um setting terapêutico o mais parecido possível com o presencial. Dito de outro modo, pela videoconferência é possível ao profissional e ao paciente interagirem por som e imagem, o que permite trocas verbais e não-verbais de modo síncrono, mesmo que essas últimas sejam de forma mais limitada, quando comparado ao atendimento presencial ${ }^{3}$.

Além disso, pesquisas têm sido realizadas considerando o processo psicoterapêutico por meio de outras tecnologias, como o uso do telefone, por exemplo $0^{9,10}$. Em específico, cabe ressaltar que em alguns países como a Áustria, no momento inicial da pandemia, o recurso telefônico foi a primeira opção para os atendimentos psicológicos, visto a falta de regulamentação no país para o uso da internet em atendimentos psicológicos online ${ }^{9}$.

Pode-se perceber que a realização das psicoterapias no contexto online, naturalmente, impõe diversos desafios aos profissionais, notadamente habituados ao contexto de atendimentos presenciais. Estudos já foram realizados anteriormente a $2020^{2,3}$, no entanto, a emergência pela oferta de serviços online durante a pandemia de COVID-19 intensificou a necessidade de investigações sobre o tema, principalmente sobre a transição entre os dois contextos terapêuticos.

Tabela 1. Informações dos artigos analisados.

\begin{tabular}{|c|c|c|c|c|}
\hline Título do artigo & Autores & $\begin{array}{l}\text { Periódico/ } \\
\text { ano de } \\
\text { publicação }\end{array}$ & Objetivo & Participantes do estudo \\
\hline $\begin{array}{l}\text { Psychotherapists' } \\
\text { attitudes toward } \\
\text { online therapy } \\
\text { during the COVID-19 } \\
\text { pandemic }\end{array}$ & $\begin{array}{c}\text { Békés, V, Aafjes- } \\
\text { van Doorn K }\end{array}$ & $\begin{array}{l}\text { J Psychother } \\
\text { Integr./2020 }\end{array}$ & $\begin{array}{l}\text { Investigar como as atitudes } \\
\text { dos psicoterapeutas em } \\
\text { relação à psicoterapia online } \\
\text { são influenciadas por suas } \\
\text { características e experiências } \\
\text { profissionais durante a } \\
\text { transição da psicoterapia } \\
\text { presencial para online na } \\
\text { pandemia. }\end{array}$ & $\begin{array}{c}\mathrm{N}=145 \\
\text { Abordagens: } \\
\text { Cognitivo comportamental; } \\
\text { Psicodinâmica; } \\
\text { Psicanalítica; } \\
\text { Humanista; } \\
\text { Sistêmica; } \\
\text { Integrativa; } \\
\text { Outras. }\end{array}$ \\
\hline $\begin{array}{l}\text { Stretching the } \\
\text { analytic frame: } \\
\text { Analytic therapists' } \\
\text { experiences with } \\
\text { remote therapy } \\
\text { during COVID-19 }\end{array}$ & $\begin{array}{c}\text { Békés V, Aafjes- } \\
\text { van Doorn } \\
\text { K, Prout TA, } \\
\text { Hoffman L }\end{array}$ & $\begin{array}{l}\text { J. Amer. Psych. } \\
\text { Assoc./2020 }\end{array}$ & $\begin{array}{l}\text { Investigar a transição de } \\
\text { psicoterapeutas analíticos } \\
\text { para a terapia online por } \\
\text { videoconferência durante a } \\
\text { pandemia, e suas experiências } \\
\text { com a terapia à distância. }\end{array}$ & $\begin{array}{l}\mathrm{N}=190 \\
\text { Abordagem: } \\
\text { Analítica. }\end{array}$ \\
\hline
\end{tabular}




\begin{tabular}{|c|c|c|c|c|}
\hline Título do artigo & Autores & $\begin{array}{l}\text { Periódico/ } \\
\text { ano de } \\
\text { publicação }\end{array}$ & Objetivo & Participantes do estudo \\
\hline $\begin{array}{l}\text { Psychotherapy } \\
\text { during COVID-19: } \\
\text { How the clinical } \\
\text { practice of italian } \\
\text { psychotherapists } \\
\text { changed during the } \\
\text { pandemic }\end{array}$ & $\begin{array}{l}\text { Boldrini T, } \\
\text { Lomoriello } \\
\text { AS, Corno FD, } \\
\text { Lingiardi V, } \\
\text { Salcuni S }\end{array}$ & $\begin{array}{c}\text { Front. } \\
\text { Psychol/2020 }\end{array}$ & $\begin{array}{l}\text { Estudar quais os fatores } \\
\text { relacionados aos } \\
\text { psicoterapeutas e sua } \\
\text { prática clínica tiveram papel } \\
\text { importante nas taxas de } \\
\text { tratamento interrompidos } \\
\text { durante o lockdown, e a } \\
\text { satisfação dos terapeutas com } \\
\text { a psicoterapia online. }\end{array}$ & $\begin{array}{c}\mathrm{N}=308 \\
\text { Abordagens: } \\
\text { Psicodinâmica; } \\
\text { Cognitivo comportamental; } \\
\text { Sistêmica; } \\
\text { Humanista; } \\
\text { Integrativa. }\end{array}$ \\
\hline $\begin{array}{l}\text { Provision of } \\
\text { psychotherapy } \\
\text { during the COVID-19 } \\
\text { pandemic among } \\
\text { Czech, German and } \\
\text { Slovak } \\
\text { psychotherapists }\end{array}$ & $\begin{array}{c}\text { Humer E, } \\
\text { Pieh C, Kuska } \\
\text { M, Barke A, } \\
\text { Doering BK, } \\
\text { Gossmann K, } \\
\text { Trnka R, Meier } \\
\text { Z, Kascakova N, } \\
\text { Tavel P, Probst T }\end{array}$ & $\begin{array}{l}\text { Int. J. Environ. } \\
\text { Res. Public } \\
\text { Health/2020 }\end{array}$ & $\begin{array}{c}\text { Investigar o medo dos } \\
\text { psicoterapeutas de serem } \\
\text { infectados com COVID-19 } \\
\text { durante a terapia presencial } \\
\text { e avaliar como a oferta de } \\
\text { psicoterapia mudou devido } \\
\text { à situação de pandemia, e se } \\
\text { havia diferenças em relação à } \\
\text { país e gênero. }\end{array}$ & $\begin{array}{c}\mathrm{N}=338 \\
\text { Não há menção a } \\
\text { abordagem dos terapeutas. }\end{array}$ \\
\hline $\begin{array}{l}\text { Psychotherapy } \\
\text { via the internet: } \\
\text { What programs do } \\
\text { psychotherapists } \\
\text { use, how well- } \\
\text { informed do they } \\
\text { feel, and what } \\
\text { are their wishes } \\
\text { for continuous } \\
\text { education? }\end{array}$ & $\begin{array}{c}\text { Humer E, } \\
\text { Stippl P, Pieh C, } \\
\text { Schimböck W, } \\
\text { Probst T }\end{array}$ & $\begin{array}{l}\text { Int. J. Environ. } \\
\text { Res. Public } \\
\text { Health/2020 }\end{array}$ & $\begin{array}{c}\text { Examinar o quão bem } \\
\text { informados se sentiam os } \\
\text { psicoterapeutas austríacos } \\
\text { sobre o uso da internet em } \\
\text { psicoterapia, quando existiu } \\
\text { necessidade de informação } \\
\text { adicional, e qual software era } \\
\text { utilizado nas sessões. }\end{array}$ & $\begin{array}{c}\mathrm{N}=1.547 \\
\text { Abordagens: } \\
\text { Psicodinâmica; } \\
\text { Humanista; } \\
\text { Sistêmica; } \\
\text { Comportamental; } \\
\text { Outras. }\end{array}$ \\
\hline $\begin{array}{l}\text { The challenges } \\
\text { and experiences of } \\
\text { psychotherapists } \\
\text { working remotely } \\
\text { during the } \\
\text { coronavirus } \\
\text { pandemic }\end{array}$ & $\begin{array}{l}\text { McBeath, AG, } \\
\text { Plock, S, Bager- } \\
\text { Charleson, S }\end{array}$ & $\begin{array}{l}\text { Couns } \\
\text { Psychother } \\
\text { Res./2020 }\end{array}$ & $\begin{array}{l}\text { Explorar as experiências e } \\
\text { desafios dos psicoterapeutas } \\
\text { que trabalham remotamente } \\
\text { durante a pandemia do novo } \\
\text { coronavírus. }\end{array}$ & $\begin{array}{c}\mathrm{N}=335 \\
\text { Abordagens: } \\
\text { Integrativa; } \\
\text { Centradas na pessoa; } \\
\text { Analítica; } \\
\text { Cognitivo comportamental; } \\
\text { Psicodinâmica; } \\
\text { Existencial; } \\
\text { Gestalt; } \\
\text { Humanista; } \\
\text { Pluralista; } \\
\text { Outras. }\end{array}$ \\
\hline $\begin{array}{l}\text { Changes in provision } \\
\text { of psychotherapy in } \\
\text { the early } \\
\text { weeks of the } \\
\text { COVID-19 lockdown } \\
\text { in Austria }\end{array}$ & $\begin{array}{c}\text { Probst, T, Stippl, } \\
\text { P, Pieh, C }\end{array}$ & $\begin{array}{l}\text { Int. J. Environ. } \\
\text { Res. Public } \\
\text { Health/2020 }\end{array}$ & $\begin{array}{c}\text { Investigar como a oferta } \\
\text { de psicoterapia mudou } \\
\text { nas primeiras semanas de } \\
\text { lockdown devido à COVID-19 } \\
\text { na Áustria e se teve diferenças } \\
\text { entre as quatro orientações } \\
\text { terapêuticas elegíveis no país } \\
\text { (psicodinâmica, humanista, } \\
\text { sistêmica e comportamental). }\end{array}$ & $\begin{array}{l}\mathrm{N}=1.547 \\
\text { Abordagens: } \\
\text { Psicodinâmica; } \\
\text { Humanista; } \\
\text { Sistêmica; } \\
\text { Comportamental; } \\
\text { Outras. }\end{array}$ \\
\hline
\end{tabular}

Um dos desafios, reportado nos estudos, diz respeito ao uso da própria tecnologia em si, e que em alguns momentos poderia ser falha, como nos casos de uma conexão de internet ruim. Essas interrupções 
ou até ausências de sinal são entendidos como fatores que tendem a prejudicar a efetividade do tratamento por, de certo modo, causar mal-entendidos entre psicoterapeutas e pacientes, o que pode comprometer a aliança terapêutica estabelecida ${ }^{6,8}$. Além disso, a necessidade de aprender novas tecnologias para ofertar os atendimentos online foi reportado. No entanto, esse aprendizado ocorreu de forma solitária, com poucas discussões com outros profissionais mais experientes ${ }^{4,6}$. Ademais, o profissional ser familiarizado com as tecnologias utilizadas, bem como aceitar o auxílio do paciente sobre o uso de recursos tecnológicos, se mostrou como um fator que contribuiu para a adesão do paciente à psicoterapia online ${ }^{8}$.

Desafios mais diversificados e expressos de forma intensa apareceram no estudo de Békés e Aafjes-van Doorn ${ }^{4}$ realizado com 145 psicólogos dos Estados Unidos, Canadá e Europa, que passaram pela mudança abrupta do contexto de atendimento presencial para online durante a pandemia de COVID-19. Os problemas técnicos e com a internet foram considerados barreiras importantes no oferecimento da psicoterapia em ambiente virtual, e o manejo desses problemas foi considerado um grande desafio para os psicoterapeutas. No entanto, aspectos relacionados ao manejo clínico e a estruturação do setting online foram prioritariamente enfatizados. Dificuldades em encontrar um espaço adequado em casa para os atendimentos, assim como se adaptar ao contexto de vida real dos pacientes, passível de maior distração, foram salientados pelos profissionais. Isso remete ao fato de que, no contexto de atendimentos psicológicos online, acaba-se colocando a necessidade de o setting ser manejado tanto pelos psicólogos quanto pelos pacientes, para garantir os princípios técnicos e éticos da Psicologia ${ }^{1}$.

Apesar dessas inseguranças iniciais, os terapeutas reportaram atitudes positivas ou neutras de seus pacientes com relação à psicoterapia online, bem como suas próprias atitudes positivas, principalmente quando passaram a se sentir mais confiantes no trabalho oferecido ${ }^{4}$. Estudos mostraram que os profissionais mais velhos e com mais experiência clínica apresentaram maior atitude positiva frente aos atendimentos online, visto que conseguiam manter a técnica psicoterapêutica e a garantia da eficácia do tratamento, apesar dos desafios no uso das tecnologias. Já para os psicoterapeutas mais jovens, os desafios estiveram atrelados ao manejo clínico na psicoterapia online, o que colocava sentimentos de ansiedade e insegurança frente a eficácia da modalidade de atendimento online oferecida ${ }^{7,8}$. Estudos prévios realizados já indicavam a eficácia do atendimento online, pontuando também as ressalvas que os terapeutas tinham com o uso dessa modalidade e a maior aceitação por parte dos pacientes ${ }^{2,3}$.

Ainda, outro desafio destacado pelos profissionais foi a adaptação ao novo ritmo da sessão no contexto online, que tendia a se diferir quando comparado ao atendimento presencial. Nesse cenário, aspectos da interação terapêutica, como dificuldades em se conectar aos pacientes e em ler suas emoções foi um desafio reportado ${ }^{4,8}$. Nesse sentido, torna-se importante o desenvolvimento de habilidades terapêuticas na condução das sessões na modalidade online, que de certo modo podem ser alcançadas através de formações específicas e de supervisão. $O$ conhecimento dos recursos tecnológicos utilizados também torna o manejo do atendimento mais assertivo ${ }^{4,5,8}$. Isso corrobora com as informações do Guia de Orientação para Profissionais de Psicologia ${ }^{1}$ que ressalta a importância de o terapeuta ter conhecimentos técnicos básicos das ferramentas que disponibiliza 
ao paciente. A apropriação desse conhecimento oferece maior segurança na construção do setting terapêutico e no aumento da confiança do terapeuta ao conduzir o atendimento online.

Por fim, se faz relevante destacar algumas limitações do presente estudo. As bases de dados pesquisadas e os descritores selecionados podem ter restringido o acesso a um número maior de artigos sobre psicoterapia online e COVID-19. Sugere-se que em estudos futuros possam ser utilizadas outras bases de dados (ex: Web of Science), de modo a ampliar a busca por material analisado. Outra sugestão é que os próximos estudos possam acessar abordagens psicoterapêuticas específicas (ex: analíticas, cognitivo comportamental e outras), a fim de averiguar se há particularidades na experiência dos profissionais e dos pacientes de acordo com a terapêutica utilizada, o que não foi possível investigar no presente estudo.

Ainda, os estudos analisados, notadamente, consideram o contexto de atendimento online do paciente adulto, sendo que pouco até então foi produzido sobre a psicoterapia online infantil e adolescente. Neste aspecto, pode-se pensar que os avanços da psicologia clínica se dão, tradicionalmente, no sentido de entender como as mudanças e novas técnicas se aplicam ao público adulto para, somente posteriormente, entender esta dinâmica em grupos diversos, como as crianças e os adolescentes, por exemplo. Com isso, tem-se ainda um grande leque de estudos que precisam ser realizados sobre efetividade, manejo e possibilidades de aplicação da psicoterapia online considerando públicos, atividades e contextos diversificados de oferta deste serviço. Os desafios frente ao manejo clínico da psicoterapia infantil e adolescente na modalidade online precisam ser debatidos, uma vez que a privacidade e o sigilo da sessão com esse público, nos atendimentos virtuais, ganham outros contornos, uma vez que o lar passa a ser, também, o ambiente psicoterapêutico.

Para encerrar, pode-se destacar que o uso das modalidades síncronas de atendimento foi predominante nos artigos analisados (ex: videoconferência, telefone), quando comparado ao uso de modalidades assíncronas (ex: mensagens de texto e e-mails). Nesse sentido, mais estudos precisam ser realizados a fim de se investigar o potencial terapêutico que os meios assíncronos podem trazer para as psicoterapias, bem como a melhor maneira de utilizá-los a fim de oferecer benefícios aos pacientes.

\section{Conclusão}

No momento atual, já passado mais de um ano do início das medidas de isolamento social, o que se sabe é que a pandemia de COVID-19, em particular no contexto brasileiro, veio acelerar a oferta de serviços psicológicos online. Apesar de não ser viável prever o contexto das psicoterapias no pós-COVID, é possível imaginar, a partir do cenário atual, que o futuro da psicologia clínica está entrelaçado aos contextos virtuais de atendimento. É evidente que a procura por psicoterapia presencial vai continuar, a fim de atender as demandas de um público que não responde ao contexto terapêutico mediado pelas TICs; mas é igualmente evidente que a busca por serviços online dificilmente irá retroceder.

Assim, especial atenção precisará ser dada a oferta de plataformas específicas para atendimentos psicológicos online, que garantam o estabelecimento de um setting confiável e efetivamente seguro ao 
paciente e ao psicoterapeuta, sem riscos ao sigilo das sessões, por exemplo. Atualmente tais plataformas já são ofertadas no contexto brasileiro, embora sem avaliações efetivas por parte do Conselho Federal de Psicologia, o que mostra que tal avanço é incipiente no cenário nacional. Além disso, o treinamento do profissional para o uso assertivo das tecnologias, bem como para o estabelecimento do contrato terapêutico no setting virtual tornam-se emergentes para que a psicoterapia aconteça e seja efetiva no contexto de atuação online. Nesse sentido, a necessidade de aprimoramento do profissional de psicologia referente ao trabalho clínico online, bem como a obrigatoriedade de abordar tal contexto de intervenção nos cursos de graduação e de pós-graduação em psicologia torna-se uma necessidade emergente.

\section{Referências}

1. Conselho Regional de Psicologia do Rio Grande do Sul. Guia de orientação para profissionais de Psicologia: atendimento on-line no contexto da COVID-19. Porto Alegre: CRPRS; 2020. Available from: https://www. crprs.org.br/publicacoes/guia-de-orientacao-para-profissionais-de-psicologia-atendimento-on-line-nocontexto-da-covid-19

2. Pieta MAM, Gomes WB. Psicoterapia pela Internet: viável ou inviável? Psicol cienc prof. 2014; 34(1), 18-31.

3. Ulkovski EP, Silva LPD, Ribeiro AB. Atendimento psicológico online: perspectivas e desafios atuais da psicoterapia. Rev Inic Cient Univ Vale Rio Verde, 2017; 7(1), 59-68.

4. Békés V, Aafjes-van Doorn K. Psychotherapists' attitudes toward online therapy during the COVID-19 pandemic. J Psychother Integr. 2020; 30(2), 238-247.

5. Humer E, Stippl P, Pieh C, Schimböck W, Probst T. Psychotherapy via the Internet: What Programs Do Psychotherapists Use, How Well-Informed Do They Feel, and What Are Their Wishes for Continuous Education?. Int J Environ Res Public Health. 2020; 17(21), 1-9.

6. Boldrini T, Lomoriello AS, Corno FD, Lingiardi V, Salcuni S. Psychotherapy During COVID-19: How the Clinical Practice of Italian Psychotherapists Changed During the Pandemic. Front Psychol. 2020; 11(0) 1-9.

7. Békés V, Aafjes-van Doorn K, Prout TA, Hoffman L. Stretching the analytic frame: analytic therapists' experiences with remote therapy during COVID-19. Am Psychoanal Assoc. 2020; 68(3), 437-446.

8. McBeath AG, Plock S, Bager-Charleson S. The challenges and experiences of psychotherapists working remotely during the coronavirus* pandemic. Couns Psychother Res. 2020; 20(0), 394-405.

9. Probst T, Stippl P, Pieh C. Changes in Provision of Psychotherapy in the Early Weeks of the COVID-19 Lockdown in Austria. Int J Environ Res Public Health. 2020; 17(11), 1-10.

10. Humer E, Pieh C, Kuska M, Barke A, Doering BK, Gossmann K, Trnka R, Meier Z, Kascakova N, Tavel P, Probst T. Provision of Psychotherapy during the COVID-19 Pandemic among Czech, German and Slovak Psychotherapists. Int J Environ Res Public Health. 2020; 17(13), 1-15.

Contribuições: Tatiele Jacques Bossi - Coleta de Dados, Redação - Preparação do original, Redação - Revisão e Edição;

Indianara Sehaparini - Coleta de Dados, Redação - Preparação do original, Redação - Revisão e Edição. 


\section{Correspondência}

Tatiele Jacques Bossi

tatielejbossi@gmail.com

Submetido em: 10/02/2021

Aceito em: 01/05/2021 